\title{
A biophysical model of the early olfactory system of hon- eybees
}

\author{
Ho Ka Chan ${ }^{1}$, Thomas Nowotny ${ }^{1}$ \\ ${ }^{1}$ School of Engineering and Informatics, University of Sussex, Falmer, Brighton, BN1 9QJ, \\ UK
}

\begin{abstract}
Experimental measurements often can only provide limited data from an animal's sensory system. In addition, they exhibit large trial-to-trial and animal-to-animal variability. These limitations pose challenges to building mathematical models intended to make biologically relevant predictions. Here, we present a mathematical model of the early olfactory system of honeybees aiming to overcome these limitations. The model generates olfactory response patterns which conform to the statistics derived from experimental data for a variety of their properties. This allows considering the full dimensionality of the sensory input space as well as avoiding overfitting the underlying data sets. Several known biological mechanisms, including processes of chemical binding and activation of receptors, and spike generation and transmission in the antennal lobe network, are incorporated in the model at a minimal level. It can therefore be used to study how experimentally observed phenomena are shaped by these underlying biophysical processes. We verified that our model can replicate some key experimental findings that were not used when building it. Given appropriate data, our model can be generalized to the early olfactory systems of other insects. It hence provides a possible framework for future numerical and analytical studies of olfactory processing in insects.
\end{abstract}

Keywords: insect olfaction, honeybees, model, biophysical

\section{Introduction}

To develop a quantitative understanding of animals' sensory systems, researchers build mathematical models based on experimental data. Unfortunately, due to limitations in experimental techniques, these data are necessarily noisy and incomplete. For example, in honeybees, responses of only around 30 of a total of 160 known types of olfactory receptor neurons (ORNs) can routinely be measured [1,2]. A typical modelling approach is to create reduced models using incomplete data. An example is the olfactory model in [3], which contains only the ORNs and corresponding glomeruli in the antennal lobe for which the data are available. However, it is unclear whether such models sufficiently relate to the biological systems they aim to describe, since the scaling of noise, synaptic efficacies and finite size network effects may change the dynamics of the system significantly. In addition, many numerical models create response patterns by directly fitting data from experiments in which specific properties of a subset of the 
systems are measured [3,4]. It cannot be expected that the predictions from these models would reflect other properties of the observed subsystem or any characteristics of the remainder of the system. It would also be unlikely for these models to be consistent with unrelated experimental data not used to build them. Yet other models are purely phenomenological [5]. In this case it is difficult to address how the processing and coding of stimuli are implemented biologically. It is, therefore, highly desirable to develop statistical models that both consider inputs representative of the full sensory input space of the animals to guard against over-fitting to limited data and at the same time incorporate relevant underlying biophysical processes to allow relating the model back to biology.

In this work, we illustrate a method for building full-size models of animals' sensory systems that extrapolates inputs from a limited subset of available experimental observations using the example of the early olfactory system of honeybees. The resulting model comprises the full number of 160 different types of ORNs as well as local neurons (LNs) and projection neurons (PNs) organized in 160 corresponding glomeruli. The ORN response patterns are generated using a set of ordinary differential equations describing the binding and activation of receptors closely related to the actual biological processes [3,4], while the response of PNs are determined by network input from ORNs and LNs using a simple rate model derived from the leaky integrate-and-fire model.

The remainder of the paper is organized as follows. In Section 2, we describe in details how our model was built. In Section 3, we show that our model reproduces key features of ORN and PN responses to continuous stimuli (3.1) and short pulses (3.2) observed in experimental work that was not considered when building the model $[9,10,13,14]$. In Section 4, we discuss the strength and limitations of our model and our plans for future work.

\section{Methods}

In our model, responses from the same type of ORNs, LNs or PNs are approximated by their ensemble average. We therefore use a single unit to represent all units of the same type. In this report, we refer to each type of a certain entity by its representatives (e.g. 20 types of ORNs will be referred to as 20 ORNs)

\subsection{Asymptotic response of model ORNs for time-invariant odor inputs at high concentration}

Asymptotic responses of 28 ORNs for time-invariant odor inputs at high concentration to 16 different odors have been measured using calcium imaging of glomeruli with bath-applied $\mathrm{Ca}^{2+}$ dyes [1]. We adopted these responses directly to form the responses of the first 28 ORNs in our model. We then generated the responses of the remaining 132 ORNs to the same 16 odors using a method inspired by [6]. The response patterns were generated from a combination of previously generated responses, including those from [1], and noise. The parameters were chosen such that the statistical distribution of the pairwise correlations of ORNs across odours in the generated responses matches that of the 28 ORNs adopted from data. The generated responses were then rescaled 
such that the mean and the variance of the responses for all receptor-odour combinations, and the mean and the variance of the variance of the responses across odours for each ORN also match.

ORN responses to chemically similar odours are correlated [7]. In our model, such correlations are quantified using the normalized Euclidean distances $d_{i j}$ between the response vectors of 2 odours $i$ and $j$, denoted by $x_{i}$ and $x_{j}$, as in [7].

$d_{i j}=\sqrt{\frac{\sum_{k}\left(x_{i k}-x_{j k}\right)^{2}}{N}}$,

where $N$ is the total number of odours in our input space and the subscript $k$ labels the different ORNs.

The responses of all previously generated ORNs were then iteratively tuned so that the Euclidean distance matrix $d$ for the generated response patterns matches that calculated from the experimental data. The tuning processes are designed to cause insignificant changes to the statistical quantities calibrated previously.

\subsection{Time series response at other concentrations}

The time series response to a single odour stimulus was generated using a set of ordinary differential equations describing the binding and activation of receptors as in [3]:

$$
\left\{\begin{array}{c}
\dot{r}=k_{-1} r_{b}-k_{1} r c^{n} \\
\dot{r_{b}}=k_{1} r c^{n}-k_{-1} r_{b}+k_{-2} r_{b}{ }^{*}-k_{2} r_{b} \\
r_{b}{ }^{*}=k_{2} r_{b}-k_{-2} r_{b}{ }^{*} \\
r+r_{b}+r_{b}{ }^{*}=r_{0}
\end{array},\right.
$$

where $k_{1}\left(k_{-1}\right)$ and $k_{2}\left(k_{-2}\right)$ are the (un)binding constants and (de)activation constants respectively, $c$ is the concentration of the odor, $n$ describes the effects of the transduction cascade, and $r, r_{b}$ and $r_{b}{ }^{*}$ are the 'effective concentration' of free, bound and activated receptors such that $r_{b}{ }^{*}$ is proportional to the excitatory conductance of the ORN. The sum of the number of receptors in different states is equal to the total number of available receptors, $r_{0}$, as described by the last equation.

Denoting $r_{b}{ }^{*}$ as the receptor response, the equilibrium response-dose relationship can be described by Hill curves when a time-invariant stimulus is applied [4]. $n$, $k_{1}\left(k_{-1}\right)$ and $k_{2}\left(k_{-2}\right)$ are partially constrained by the parameters in the Hill curves, which are statistically sampled in accordance to experimental observations in [8]. To deal with the remaining degrees of freedom, we took into account the typical timescale of dynamics in $\mathrm{AL}$ responses measured experimentally $[9,10]$.

\subsection{Obtaining the instantaneous firing rate of neurons}

To obtain the firing rate of the ORNs from its input conductances (which are assumed to be proportional to $r_{b}{ }^{*}$; see 2.2), the dynamics of a neuron is approximated by the conductance-based leaky integrate-and-fire model with adaptation as shown in (3). 


$$
\begin{aligned}
& \tau_{\text {eff }}(t) \frac{d V}{d t}=-V+R I_{\text {eff }}(\mathrm{t})-R I_{\text {adapt }}(t) \\
& \tau_{\text {adapt }} \frac{d I_{\text {adapt }}(t)}{d t}=-I_{\text {adapt }}(t) \\
& I_{\text {adapt }}=I_{\text {adapt }}^{\max } \text { at } t=t_{f}
\end{aligned}
$$

Detailed descriptions of the parameters in (3) can be found in [11]. We then adopted the adiabatic approximation by considering the input to be quasi-time-invariant on the time scale of neuronal firing, such that $\tau_{\text {eff }}(t)$ and $I_{\text {eff }}(\mathrm{t})$ are taken to be constant. With the additional assumption of noise-free input and setting $t_{f}=0$, the membrane potential before the next firing event can be obtained analytically as follows:

$V=V_{\text {reset }} e^{\frac{-t}{\tau_{\text {eff }}}}+I_{\text {eff }}\left(1-e^{-\frac{t}{\tau_{\text {eff }}}}\right)-\frac{\tau_{\text {adapt }} \max _{\text {adapt }}^{\text {max }}}{\tau_{\text {adapt }}-\tau_{\text {eff }}}\left(e^{-\frac{t}{\tau_{\text {adapt }}}}-e^{-\frac{t}{\tau_{\text {eff }}}}\right)$,

where $V_{\text {reset }}$ is the reset potential after the neuron has fired. The instantaneous firing rate of the neuron can then be obtained using:

$$
v=\frac{1}{t_{\text {thres }}}+t_{\text {refract }},
$$

where $t_{\text {thres }}$ is the time when $V=V_{\text {th }}$, which is to be obtained numerically, and $t_{\text {refract }}$ is the absolute refractory period. Note that in (4) and (5), we have set $R=1$ by absorbing it into $I_{\text {adapt }}^{\max }$ and other variables.

We chose $I_{\text {adapt }}^{\max }=I_{\text {adapt }}^{\text {base }} \sqrt{r_{b}{ }^{*}}$ for ORNs, and $I_{\text {adapt }}^{\max }=I_{\text {adapt }}^{\text {base }} \sqrt{v_{\text {pre }}}$ for PNs and LNs, where $I_{\text {adapt }}^{\text {base }}$ is a constant and $v_{\text {pre }}$ is the firing rate of the corresponding units in the previous iteration. However, qualitatively similar results can be obtained by assuming $I_{\text {adapt }}^{\max }$ to be constant (results not shown).

\subsection{Generating PN responses}

In our model, ORNs provide excitatory input to PNs and LNs. Both receive excitatory input from the ORNs of their own glomerulus, with uniform connectivity, as well as inhibitory input from LNs of all other glomeruli. This is illustrated in Fig. 1.

To be consistent with the findings in [12], for any pair of glomeruli $i$ and $j$, the connectivity between the PN in glomerulus $i$ and the $\mathrm{LN}$ in glomerulus $j$ is weighted by $w_{i j}$, which is based on the correlations $\rho_{i j}$ between the corresponding ORN responses across different odours,

$w_{i j}=\left(1-\delta_{i j}\right)\left[w_{0}+H\left(\rho_{i j}\right) \times \rho_{i j} w_{\mathrm{corr}}\right]$,

where $\delta_{i j}$ is the Kronecker delta function, $H$ is the Heaviside step function, $w_{0}$ and $w_{\text {corr }}$ are normal distributed random variables. $\rho_{i j}$ is the Pearson correlation between the conductance of ORNs $i$ and $j$ as obtained in 2.1, across odours. 


$$
\rho_{i j}=\frac{\operatorname{Cov}\left(x^{T}{ }_{i} x^{T}{ }_{j}\right)}{\sigma\left(x^{T}{ }_{i}\right) \sigma\left(x^{T}{ }_{j}\right)}
$$

The firing rate of LNs and PNs are calculated by (4) and (5). The calculations are iterated several times to allow the system to settle into a steady state. This effectively assumes that any oscillations in PN activity are negligible and thus ignored.

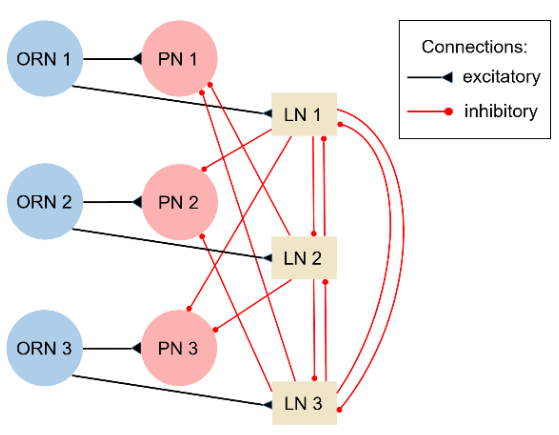

Fig. 1. Illustration of part of the model AL network.

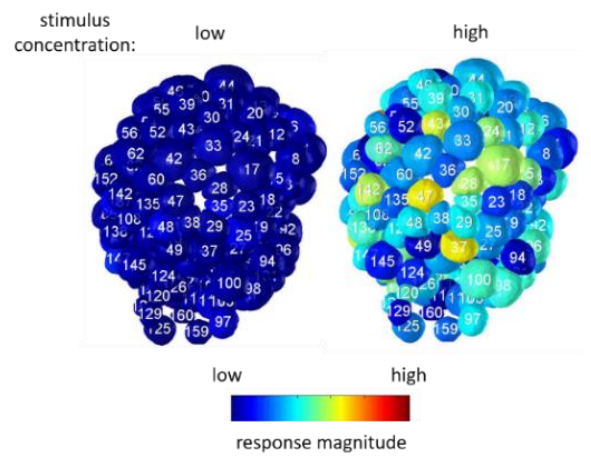

Fig. 2. The model predicted asymptotic ORN responses to 1-hexanol at low $\left(c=10^{-4} M\right.$, left) and high $\left(c=10^{-1} M\right.$, right $)$ concentrations.

\section{Results}

\subsection{Response to continuous stimuli}

Using the methods described in Section 2, we built a model that can generate asymptotic and time series responses of all 160 glomeruli to 16 different odours. We first tested the ORN responses generated by our model with continuous stimuli. As an example, the asymptotic ORN responses to 1-hexanol at two different concentrations is shown in Fig. 2. At low concentration, most ORNs are quiescent; while at high concentration, almost all ORNs are activated to some degree.

We next compared the results of PN responses obtained from our model to $\mathrm{Ca}^{2+}$ imaging data with back-filled PNs [13]. Previous studies [2] have shown that unlike ORNs, which responses almost always increase with dose, PN responses display a variety of relationships with dose due to inhibition from LNs. In Figure 3, we divide the response-dose relationships into 4 different types: "inactivated" where PNs show no or very weak responses to stimuli at any dose, "decrease" ("increase") where responses decrease (increase) with dose and "other" where responses are independent of or display non-monotonic relationships with dose, and show that the statistical distribution of each type of response-dose relationship observed in the model PNs matches very well to experimental PN data in [13]. This suggests that our network model can reasonably capture the effects of LN inhibition. 


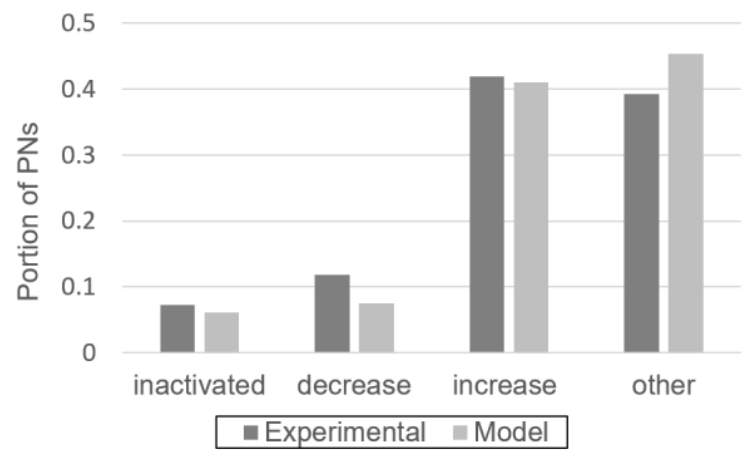

Fig. 3. Statistical distribution of different kinds of response-dose relationships observed in model PN responses (right bars) and experimental PN data from [7] (left bars).

Finally, we compared the ORN and PN responses from our model. Figure 4 (left) shows the probability distribution of pairwise correlations between experimental and model ORN and PN responses across odours. While the model ORN responses are highly correlated as in the corresponding experimental ORN data, the model PN responses are mostly uncorrelated, with the peak of the probability around zero correlation. Our model results for both ORNs and PNs show a good fit to their experimental counterparts in $[1,13]$ qualitatively, even though the model was only fitted to ORN data. Please note that the 'model responses' correspond to firing rate, which may not map directly to $\mathrm{Ca}^{2+}$ imaging data. Figure 4 (right) shows that the correlation between our model ORN and PN response patterns to different odours centred around 0.7, which agrees with experimental data [14], which shows that the correlation between ORN and AL activity is around 0.6-0.7.

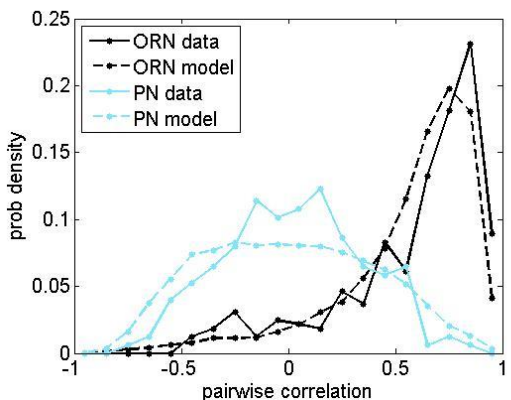

Fig. 4. Statistical distribution for pairwise correlations for different ORNs $(c=1)$ and PNs $(c=0.1)$ across response patterns, observed in $\mathrm{Ca}^{2+}$ imaging experiments $[1,13]$ and our model.

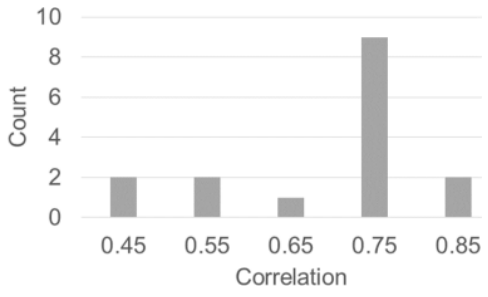

Fig. 5. Statistical distribution of the pairwise correlations between the overall ORN and PN response for different odour stimuli.

\subsection{Response to short pulses}

In addition to continuous stimuli, we further tested our model with stimuli consisting of short pulses. Figure 6 shows the average ORN responses to 1-hexanol to square 
pulses of $2 \mathrm{~ms}$ with different inter-pulse intervals and compares them to those measured by electro-antennogram recordings [9]. The model responses exhibit all relevant features observed in the experimental data except that the time scale of response latency is smaller. This can be explained by the lack of temporal filtering of input conductance to output spiking in our rate model.

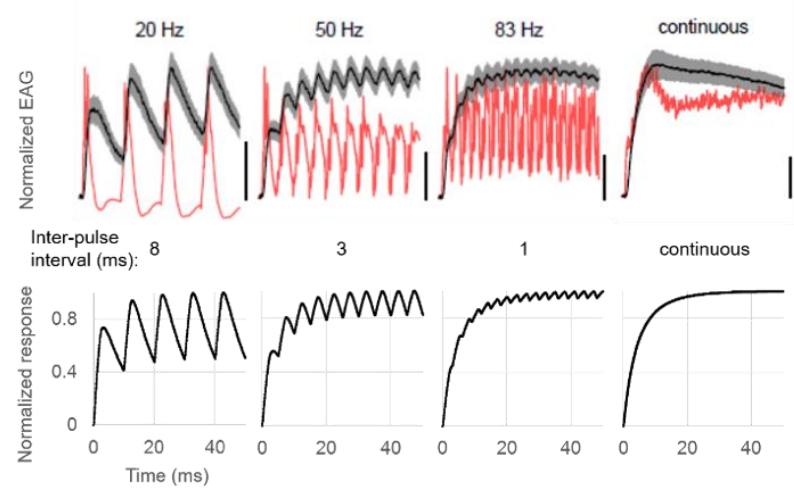

Fig. 6. ORN responses to pulsed and constant stimuli (top, red line) as measured by electroantennogram recordings [9] (top, black line) and the average normalized ORN responses to similar stimuli (1-hexanol at concentration $0.1 \mathrm{M}$ in square pulses of $2 \mathrm{~ms}$ ) predicted by our model (bottom).

\section{Discussion}

In this work, we demonstrated how we built a mathematical model of the early olfactory system of honeybees using severely limited data. We were able to generate ORN response patterns using a very simple biophysical model of receptors and generate responses of other parts of the system using a network model mimicking the widely accepted structure of honeybees' antennal lobe. The response to different types of stimuli predicted by our model matches that obtained from experimental measurements very well on the statistical level. Our methods make full use of our knowledge about honeybees' olfactory system and require extremely small amounts of computation. They are general enough such that, given appropriate data, they could be readily applied to model olfactory systems of other insects, or, with slight modifications to the receptor dynamics and the neural network, to model other sensory systems.

Unlike model in previous work, the model we developed here is not directly fitted to data, but to their overall statistics $[3,4]$. This allows us to study the statistical responses to different types of stimuli, with the trade-off that a generated model glomerulus may not correspond to any particular glomerulus in a honeybee. On the other hand, it allows us to model the full number of 160 glomeruli, even though data normally are only acquired from around 30 . Biologically, responses of a sensory unit to a stimulus may differ greatly among individual animals due to factors like genetic heterogeneity and past learning experience. Moreover, many coding strategies are believed to be 
based on ensemble behaviours $[2,3,14]$. Therefore, we believe that reproducing the statistics of observed data, rather than detailed measurements of individual cells, is more useful when modelling sensory systems of animals in most circumstances.

In this work, we used a simple firing rate model to describe the input-output relationship of neurons. A major benefit is that it is analytically tractable and drastically reduces computational costs. We have shown that most features in experimental findings can be reproduced with such a simplified model. In the model, the major approximations are the adiabatic approximation in the firing rate and the absence of input noise. The lack of temporal filters for output spiking in the model leads to an overestimation in the sensitivity of neurons to input fluctuations [15]. However, in this context, such effects are alleviated by the additional temporal filters in the process of binding and activation of receptors, as described in (3), which smoothen $I_{\text {eff }}$ to some extent even if the stimulus intensity fluctuates rapidly. The absence of input noise also has little consequence since most of the neurons are mean-driven. Mathematical formalisms $[15,16]$ have been developed such that the mean firing rate can be computed without having to make the above-mentioned approximations, but this is beyond the scope of this work.

Our future work will involve analysing the biophysical processes which give rise to the above results [17]. We also aim to modify (3) to study the olfactory response to mixtures. Preliminary results [17] suggest that receptor dynamics account for differences in olfactory processing between complex mixtures and simpler compounds, which may lead to more efficient and robust coding for complex mixtures.

\section{References}

1. Galizia, C. G., Sachse, S., Rappert, A., \& Menzel, R.: The glomerular code for odor representation is species specific in the honeybee Apis mellifera. Nature Neuroscience 2(5), 473-478 (1999).

2. Sachse, S., \& Galizia, C. G.: The coding of odour-intensity in the honeybee antennal lobe: Local computation optimizes odour representation. European Journal of Neuroscience 18(8), 2119-2132 (2003).

3. Nowotny, T., Stierle, J. S., Galizia, C. G., \& Szyszka, P.: Data-driven honeybee antennal lobe model suggests how stimulus-onset asynchrony can aid odour segregation. Brain Research, 1536, 119-134 (2013).

4. Rospars, J.-P., Lansky, P., Chaput, M., \& Duchamp-Viret, P.: Competitive and noncompetitive odorant interactions in the early neural coding of odorant mixtures. The Journal of Neuroscience, 28(10), 2659-2666 (2008).

5. Luo, S. X., Axel, R., \& Abbott, L. F.: Generating sparse and selective third-order responses in the olfactory system of the fly. Proceedings of the National Academy of Sciences of USA 107(23), 10713-10718 (2010).

6. Haenicke, J.: Modeling insect inspired mechanisms of neural and behavioral plasticity. [PhD thesis]. Berlin: Freie Universität Berlin (2015).

7. Carcaud, J., Hill, T., Giurfa, M., \& Sandoz, J.: Differential coding by two olfactory subsystems in the honeybee brain. Journal of Neurophysiology 108, 1106-1121 (2012). 
8. Grémiaux, A., Nowotny, T., Martinez, D., Lucas, P., \& Rospars, J.-P.: Modelling the signal delivered by a population of first-order neurons in a moth olfactory system. Brain Research 1434, 123-35 (2012).

9. Szyszka, P., Gerkin, R. C., Galizia, C. G., \& Smith, B. H.: High-speed odor transduction and pulse tracking by insect olfactory receptor neurons. Proceedings of the National Academy of Sciences of USA 111(47), 16925-30 (2014).

10. Szyszka, P., Stierle, J. S., Biergans, S., \& Galizia, C. G.: The speed of smell: Odor-object segregation within milliseconds. PLoS ONE 7(4), 4-7 (2012).

11. Chan, H. K., Yang, D.-P., Zhou, C., \& Nowotny, T.: Burst Firing Enhances Neural Output Correlation. Frontiers in Computational Neuroscience 10(May), 1-12 (2016).

12. Linster, C., Sachse, S., \& Galizia, C. G.: Computational modeling suggests that response properties rather than spatial position determine connectivity between olfactory glomeruli. Journal of Neurophysiology 93(6), 3410-3417 (2005).

13. Ditzen, M.: Odor concentration and identity coding in the antennal lobe of the honeybee Apis mellifera. [PhD thesis]. Berlin: Freie Universität Berlin (2005).

14. Deisig, N., Giurfa, M., Sandoz, J. C., Deisig, N., Giurfa, M., \& Sandoz, J. C.: Antennal Lobe Processing Increases Separability of Odor Mixture Representations in the Honeybee. The Journal of Physiology 103, 2185-2194 (2010)

15. Ostojic, S., \& Brunel, N.: From Spiking Neuron Models to Linear-Nonlinear Models. PLoS Computational Biology, 7(1), 1-16 (2011).

16. Chan, H. K., \& Nowotny, T.: Firing probability for a noisy leaky integrate-and-fire neuron receiving arbitrary external currents. Poster presented at the $3^{\text {rd }}$ International Conference on Mathematical Neuroscience. Boulder, CO, USA (2017).

17. Chan, H. K., \& Nowotny, T.: Mixture are more salient stimuli in olfaction. bioRxiv 163238 (2017). doi: https://doi.org/10.1101/163238 